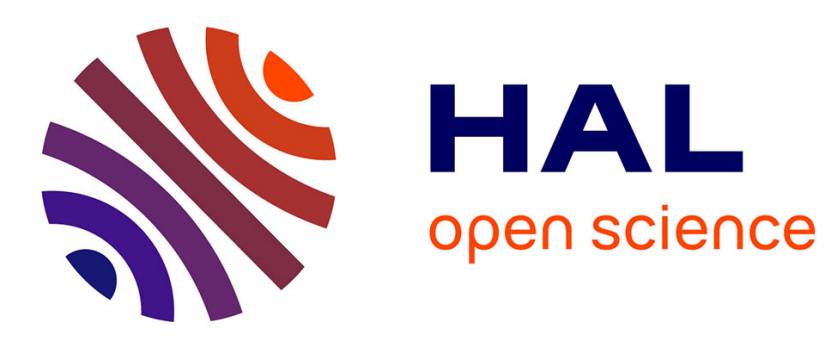

\title{
La négociation du temps de travail et les composantes du référentiel temporel
}

Jens Thoemmes, Gilbert de Terssac

\section{To cite this version:}

Jens Thoemmes, Gilbert de Terssac. La négociation du temps de travail et les composantes du référentiel temporel. Loisir et Société / Society and Leisure, 1997, 20 (1), pp.51-72. 10.1080/07053436.1997.10715537. hal-00933571v2

\section{HAL Id: hal-00933571 \\ https://hal.science/hal-00933571v2}

Submitted on 3 Aug 2014

HAL is a multi-disciplinary open access archive for the deposit and dissemination of scientific research documents, whether they are published or not. The documents may come from teaching and research institutions in France or abroad, or from public or private research centers.
L'archive ouverte pluridisciplinaire HAL, est destinée au dépôt et à la diffusion de documents scientifiques de niveau recherche, publiés ou non, émanant des établissements d'enseignement et de recherche français ou étrangers, des laboratoires publics ou privés. 
Thoemmes J., Terssac G. De, 1997, « La négociation du temps de travail et les composantes du référentiel temporel », Loisir et Société, 20, 1, p. 51-72.

\section{Jens Thoemmes et Gilbert de Terssac}

\section{La négociation du temps de travail et les composantes du référentiel temporel}

\section{Le temps de travail: de quoi parle-t-on?}

Le temps de travail est une notion complexe, car elle renvoie à des pratiques multiples et à des conceptions différentes. Tout d'abord les réalités que cette notion est censée codifier lui échappe, puisqu'elle est souvent réservée pour désigner les activités professionnelles, comme si le travail était limité au temps réservé par le salarié à son employeur. Ensuite cette notion est souvent utilisée pour désigner un temps objectif, répétitif, séquentiel, discontinu, linéaire, mesurable, quantitatif, comme si le temps subjectif, qualitatif, multiple, hétérogène constituait une autre réalité extérieure au temps de travail. Enfin le temps de travail est souvent référé à une conception unique, universelle, rationnelle, abstraite, entièrement orientée vers la performance, extérieure aux sujets et aux sociétés.

Néanmoins, les approches inscrites dans la tradition sociologique et exprimées en termes de temps sociaux (Sue, 1994), ou de temporalités sociales (Mercure, 1995) nous permettent de penser ensemble des réalités différentes et surtout d'analyser l'ordre temporel comme une construction sociale. Pour Sue (1994), il faut entendre par temps sociaux: " les grandes catégories ou blocs de temps qu'une société se donne et se représente pour désigner, articuler, rythmer et coordonner les principales activités sociales auxquelles elle accorde une importance particulière. Les grands temps sociaux ou blocs de temps se décomposent généralement aujourd'hui en temps de travail, temps de l'éducation, temps familial, temps libre ». Cette perspective relative à la découverte des temps sociaux se retrouve dans les travaux de Mercure (1995). «Par temporalités sociales il faut entendre la réalité des temps vécus par les groupes, c'est à dire la multiplicité des conduites temporelles et des représentations du temps liées à la diversité des situations sociales et de modes d'activités dans le temps » (Mercure, 1995). Selon cet auteur les «temporalités sociales » ne peuvent être analysées du seul point de vue de la répartition quantitative des activités dans le temps. Il faut y ajouter 
d'une part, la caractérisation de la multiplicité des temps sociaux, c'est à dire la diversité des temps vécus selon les individus, les groupes et les situations; il faut ajouter d'autre part, la caractérisation des rythmes sociaux, c'est à dire la régularité et la discontinuité des événements, la cadence et l'allure de nos activités. Dans cette manière de voir les temps sociaux, il devient possible de voir ensemble le temps des individus et des organisations, le temps des groupes sociaux et des institutions, le temps subjectif et le temps objectif. La tentation d'opposer ces deux réalités repose sur une séparation entre le cadre temporel et les activités sociales, sur une opposition qui est traitée dans un rapport soit de subordination des activités sociales aux temps produits par les institutions, soit de détermination du cadre temporel par le seul jeu des interactions sociales. Il nous semble préférable d'adopter une posture en terme de processus permettant d'expliciter la diversité, voir l'hétérogénéité des pratiques temporelles en référence aux manières de voir le temps et de concevoir l'ordre et le changement dans nos sociétés. Dans cette perspective, les conceptions du temps, entendues comme configurations arbitraires inscrites dans la culture de chaque société, ne constituent-elles pas en quelque sorte l'ombre portée des pratiques temporelles? De manière symétrique la variabilité et la complexité des pratiques temporelles ne s'éclairent-elles pas en fonction des constructions culturelles de chaque société?

Notre recherche sur le temps de travail est inscrite dans ce double questionnement: comment saisir la diversité des pratiques temporelles en relation avec les représentations sociales du temps? Comment penser ensemble les «modes d'activités dans le temps » (Mercure, 1995) et la diversité des situations sociales et des cadres temporels?

Le temps de travail constitue dans la perspective esquissée un champ de recherche particulièrement intéressant. Premièrement, parce que le temps de travail institue une dissociation entre d'un côté le temps de la production des biens et services qui est un temps abstrait, vide de contenu et mesuré de plus en plus rigoureusement et de l'autre, le temps vécu, celui de la vie quotidienne, non uniforme et concret. Cette dissociation entre de temps sociaux dissemblables pose le problème de leur « concordance » (Supiot, 1995), de leur «synchronisation et de leur harmonisation à l'intérieur du tissu social» (Mercure, 1995). Deuxièmement, parce que le temps de travail institue une séparation entre la vie de travail dans laquelle le temps constitue la mesure des activités professionnelles et la vie hors travail. Cette séparation constitue, comme le souligne Naville (1969), un enjeu social extrêmement important, puisqu'elle fragmente la vie sociale en deux, repartit les activités selon les durées et horaires, rythme le déroulement des activités. Le temps de travail définit le cadre des activités sociales; il inscrit les activités dans le temps et il rythme le 
passage d'une activité à l'autre. Le temps de travail nous semble bien contribuer à exprimer l'existence de ce que Pronovost (1996) appelle des « points repères dans le temps, des marqueurs du temps ».

Notre recherche sur le temps de travail vise à comprendre comment les individus «s'arrangent» pour produire des compromis temporels dans ce climat de «discordance des temps », de dysharmonie entre les temps multiples, de distorsions voire de rupture entre les temps hétérogènes. L'hypothèse générale est que les arrangements temporels auxquels les acteurs procèdent sont des constructions sociales en même temps que leur résultat. Autrement dit, l'arrangement temporel est d'abord entendu comme cadre temporel des activités, produit à coup de confrontations, de négociations et de conflits: c'est pourquoi nous proposons d'étudier les pratiques temporelles au travers de la nature et du mode de construction négociée des arrangements temporels (première partie). Mais l'arrangement temporel est aussi une traduction des représentations temporelles: c'est pourquoi nous proposons d'étudier les conceptions du temps qui jalonnent ces pratiques telle qu'elles sont exprimées par les responsables des organisations syndicales (seconde partie) et que nous appellerons les référentiels temporels (troisième partie).

Notre approche considère que le temps de travail n'est pas un cadre préexistant aux pratiques sociales et déterminant totalement leur déroulement, mais un lieu de conflit de rationalités du fait de la double dissociation qu'il institue: d'une part entre le temps du calcul ou de la production d'un côté et le temps vécu de la vie quotidienne; d'autre part entre le temps des activités professionnelles et le temps des activités nonprofessionnelles.

\section{Les pratiques de construction du cadre temporel de travail}

Le cadre temporel de travail n'est pas décrété, mais construit au travers de la négociation du temps de travail. Puisque le temps de travail est au centre d'un conflit de rationalités, (celle qui régit l'ordre de la production et celle qui régit l'ordre des activités professionnelles et non-professionnelles de la vie quotidienne), il convient de s'interroger en quoi la négociation peut apporter sa contribution à la résolution de ce conflit de rationalités. Le temps de travail comme objet de négociation est un fait récent en France qui traduit d'un côté des modes d'intervention de l'Etat différents et d'un autre côté l'ouverture à la négociation d'un espace

précédemment «verrouillé » et la recherche d'une mobilisation des partenaires sociaux pour produire des arrangements temporels pertinents et légitimes. 
Il s'agit de comprendre plus précisément en quoi la négociation du temps de travail, qui combine un mode d'intervention spécifique de l'Etat et la mobilisation des partenaires sociaux, peut conduire à la production d'un cadre temporel de travail légitime pour chacune des parties engagées dans la négociation. La question n'est pas simplement de savoir si les partenaires sociaux sont capables de coopérer, alors que le temps de travail les divise et les oppose. Il s'agit de comprendre aussi les conditions de la négociation elle-même et avant tout l'objet de la négociation. N'y a-t-il pas un paradoxe à supposer que la négociation du temps de travail est possible, alors que la réalité négociée est plurielle et que ces réalités négociés sont hétérogènes entre elles? Le temps abstrait de la production, vide de contenu peut-il être confronté aux divers temps entre lesquels les individus partagent leur existence? Autrement dit l'analyse de la négociation du temps de travail ne doit pas présupposer son objet, ni le tenir pour acquis: l'analyse doit le construire. C'est pourquoi nous proposons d'utiliser la notion « d'arrangement temporel »: d'un côté cette notion désigne un compromis dont le résultat est fragile; de l'autre, elle désigne un accord non seulement sur une solution locale, mais sur la réalité du temps qui est négociée.

Nous analyserons dans un premier temps, la négociation du temps de travail au travers d'une analyse de l'évolution des dispositifs et des pratiques (2.1). Dans un deuxième temps, nous chercherons à caractériser la dynamique de construction des arrangements temporels (2.2).

\subsection{La négociation du temps de travail: dispositifs et pratiques}

\subsection{A Des dispositifs juridiques orientés vers la flexibilité de la production}

La rationalité dominante des dispositifs de négociation du temps de travail concerne de 1980 à 1996, l'ordre de la production.

Nos travaux antérieurs menés entre juristes ${ }^{1}$ et sociologues ${ }^{2}$, notamment dans le cadre de l'évaluation de la loi quinquennale, ont montré l'évolution de la manière de traiter le temps de travail.

En France, le temps de travail a longtemps été du domaine de l'intervention de l'Etat : la législation de 1936 était fondée sur la définition d'une durée légale du travail, considérée dans le cadre de la semaine, comme une norme égalitaire fixée de façon réglementaire, donc non négociée. La définition concrète du temps de

\footnotetext{
${ }^{1}$ Equipe animée par ML Morin (LIRHE, CNRS)

${ }^{2}$ Equipe animée par G de Terssac (CERTOP, CNRS)
} 
travail était du ressort du pouvoir du chef d'entreprise. De nombreuses transformations de ce cadre tutélaire ont été apportées à partir des années 1980, pour améliorer les conditions de travail, pour améliorer la réactivité des entreprises ou pour lutter contre le chômage. Elles ont pour conséquence de modifier la nature et le sens des questions posées par la réduction du temps de travail, qui devient une question qui n'est plus posée pour elle-même, mais dans l'espace de la flexibilité du temps de travail. Les différentes lois qui vont être promulguées au cours des 15 dernières années (1981-1996) ont en commun, moins d'imposer des normes temporelles, que d'ouvrir des espaces de négociations par le jeu d'un transfert de compétences de la loi vers les partenaires sociaux ou vers les parties prenantes du contrat de travail (employeurs et salariés) : la flexibilité temporelle devient la contrepartie de toute réduction du temps de travail. De plus, la durée conventionnelle est fixée par les entreprises qui se voient dotées de marges de manoeuvre accrues et d'une plus grande souplesse pour construire des formes d'aménagement variées.

Ces transformations ont été développées pour l'essentiel depuis les années 1980. On peut les présenter comme un changement profond dans la régulation temporelle (Morin et al. 1996): d'un côté, on assiste à un changement du mode de régulation et d'un autre côté à un changement des règles de l'organisation temporelle.

La première transformation concerne le mode de régulation du temps de travail : Le temps de travail, objet de négociation. L'accord interprofessionnel du 17 juillet 1981 ouvre la voie d'un changement du mode de régulation : il accepte le principe d'une réduction de la durée du travail (réduction de la durée légale, allongement des congés payés etc.) contre un assouplissement des règles d'aménagement du temps de travail (modulation, équipes de fin de semaine, etc.). Le temps de travail est désormais traité comme une variable d'ajustement entre la logique sociale tournée vers la réduction de la durée du travail et la logique économique tournée vers l'amélioration de la compétitivité des entreprises.

La deuxième transformation concerne l'apparition de nouvelles règles temporelles :

Certes, l'ordonnance du 16 janvier 1982 opère le passage de 40 à $39 \mathrm{~h}$ et de $35 \mathrm{~h}$ pour le travail continu : la durée légale du travail est réduite et la cinquième semaine de congé est accordée ; mais toute réduction s'entend désormais en contrepartie d'un aménagement du temps de travail, qui est en fait une remise en cause du cadre normatif. L'autre volet concerne la possibilité de déroger conventionnellement aux décrets pour assouplir le cadre normatif en contrepartie de la réduction de la durée : l'ordonnance (a) introduit la possibilité de faire varier la durée hebdomadaire, à condition qu'en moyenne sur l'année, celle-ci soit égale à 
39h. (Modulation type I), (b) permet de nouvelles formes d'aménagement du temps de travail, qui déroge à la règle de l'horaire collectif et du repos dominical, comme la mise en place d'équipes de fin de semaine et le travail par roulement, maintenant autorisés, (c) et plus généralement remet en cause la répartition de la durée du travail dans le cadre de la semaine.

Dès 1984, le protocole d'accord du 16 décembre relie les questions de temps de travail à l'emploi. La loi du 28 février 1986 permettra (a) la répartition de la durée du travail sur l'année, sans paiement de la majoration des heures effectuées, dans les limites des variations de la durée hebdomadaire (modulation type II), (b) la réduction de la durée moyenne de travail et (c), la possibilité d'échanger le paiement des heures supplémentaires contre du repos. La loi du 19 juin 1987 admet des dérogations à l'interdiction du travail de nuit pour les femmes.

La loi quinquennale du 20 décembre 1993 poursuit à cet égard le mouvement engagé depuis l'ordonnance du 16 novembre 1982 qui vise à flexibiliser le temps de travail, plus précisément à favoriser l'adoption négociée « d'arrangements temporels » diversifiés, (modulation type III et temps partiel annualisé). La loi du 11 juin 1996 veut réactiver la relation entre la réduction du temps de travail et l'emploi par un renforcement du dispositif d'allégement des cotisations patronales de sécurité sociale.

\subsection{B Les négociations}

Ces règles juridiques témoignent de l'orientation du cadre légal vers une flexibilité de la production. La négociation du temps de travail confirme-t-elle cette perspective?

Notre étude des accords du temps de travail dans une région française porte sur 768 accords signés entre 1984 et 1995. Nous avons étudié, (en collaboration avec la Direction Régionale du Travail et de l'Emploi, DRTE) ces accords et nous avons montré l'existence d'une pluralité de temps qui interviennent dans la négociation. Par une méthode de classifications (nuées dynamique), nous avons mis en évidence 6 classes d'accords, reposant chacune d'elle sur des corrélations significatives entre les thèmes du temps de travail et la classe respective. Nous avons interprété et intitulé ces classes : le temps des congés, le temps confirmé (reconduction d'accords antérieurs), le temps des marchés (modulation), le temps individualisé 
(temps partiel et horaires variables), le temps échangé (réduction de la durée contre aménagement du temps de travail), le temps des machines (les horaires et notamment le travail en équipes) ${ }^{3}$,

Sur l'ensemble des 768 accords (1984-1995) nous avons isolé les 366 accords permettant d'explorer la négociation du point de vue de ses pivots structurants: réduction de la durée du travail, modulationannualisation, temps partiel et les horaires.

Notre analyse distingue des taux de refus de signature selon les organisations syndicales. Le taux de refus se rapporte exclusivement aux accords signés par l'organisation respective. ${ }^{4}$ Dans l'ensemble nous constatons une approbation forte. La CFTC (2\% de refus) et la CGC (3\%) signent quasi systématiquement. La CFDT et FO refusent de signer un accord sur 10 (9\%, 11\%), alors que la CGT en refuse un sur 4 (24\%).

Une autre perspective se dégage par les thèmes présents dans les accords. Nous pouvons constater que les « équipes de fin de semaine » sont les thèmes qui atteignent le refus de signature (18\%) le plus important, toutes organisations confondues. Le travail de nuit est refusé en moyenne dans $15 \%$ des cas (un accord sur 7). Pendant que un accord sur 10 portant sur le travail à temps partiel ou/et la modulation n'est pas signé (11\%), les syndicats limitent leur refus pour la réduction de la durée du travail à $8 \%$ (10 accords refusés sur 132).

Trois groupes d'acteurs syndicaux se dessinent dans ces résultats.

1) La CGT pratique plus couramment le refus de la signature : $36 \%$ de refus pour les équipes de fin de semaines et $30 \%$ pour le temps partiel.

2) FO et la CFDT refusent ponctuellement les accords: FO refuse davantage le travail de nuit $(21 \%$ des accords) et la CFDT qui signe moins sur les équipes de fin de semaine ( $22 \%$ des accords)

3) Enfin la CFTC et la CGC ne refusent que quelques accords très localisés.

\footnotetext{
3 Thoemmes J., Terssac (de) G. (1995).- La construction des arrangements temporels: Une étude de cas sur 11 ans, Sixième Séminaire International sur le Temps de Travail (SITT) à Blankenberge, Belgique, 9 p.

${ }^{4}$ Les pourcentages de refus d'accords doivent être maniés avec précaution en les relatant au nombre d'accord effectivement refusé.
} 
Figure 1 : taux de refus de la signature d'accord pour 4 pivots structurants de la négociation du temps de travail selon l'organisation syndicale $(1984-95)^{5},(n=366$ accords).

\begin{tabular}{|l|l|l|l|l|l|l|l|}
\hline & $\begin{array}{l}\text { Taux de refus } \\
\text { nombre d'accords } \\
\text { par thème }\end{array}$ & CGT & FO & CFDT & CFTC & CGC & $\Sigma$ \\
\hline $\mathbf{1}$ & durée (réduction) & $\mathbf{1 5 \%}$ & $\mathbf{3 \%}$ & $\mathbf{7 \%}$ & $\mathbf{1 3 \%}$ & $\mathbf{0 \%}$ & $\mathbf{8 \%}$ \\
\hline & 61 & $6 / 39$ & $1 / 35$ & $2 / 30$ & $1 / 8$ & $0 / 20$ & $10 / 132$ \\
\hline & & & & & & & \\
\hline $\mathbf{2}$ & $\begin{array}{l}\text { modulation- } \\
\text { annualisation }\end{array}$ & $\mathbf{2 5 \%}$ & $\mathbf{8 \%}$ & $\mathbf{4 \%}$ & $\mathbf{0 \%}$ & $\mathbf{4 \%}$ & $\mathbf{1 1 \%}$ \\
\hline & 98 & $15 / 60$ & $3 / 40$ & $2 / 49$ & $0 / 11$ & $1 / 26$ & $21 / 186$ \\
\hline & & & & & & & \\
\hline $\mathbf{3}$ & temps partiel & $\mathbf{3 0 \%}$ & $\mathbf{4 \%}$ & $\mathbf{1 0 \%}$ & $\mathbf{0 \%}$ & $\mathbf{6 \%}$ & $\mathbf{1 1 \%}$ \\
\hline & 49 & $8 / 27$ & $1 / 25$ & $3 / 30$ & $0 / 16$ & $1 / 16$ & $13 / 114$ \\
\hline & & & & & & & \\
\hline $\mathbf{4 . 1}$ & nuits, équipes & $\mathbf{2 1 \%}$ & $\mathbf{1 0 \%}$ & $\mathbf{2 1 \%}$ & $\mathbf{0 \%}$ & $\mathbf{5 \%}$ & $\mathbf{1 5 \%}$ \\
\hline & 125 & $19 / 90$ & $7 / 68$ & $14 / 66$ & $0 / 16$ & $2 / 44$ & $42 / 284$ \\
\hline & & & & & & & \\
\hline $\mathbf{4 . 2}$ & fin de semaine & $\mathbf{3 6 \%}$ & $\mathbf{2 2 \%}$ & $\mathbf{5 \%}$ & $\mathbf{0 \%}$ & $\mathbf{0 \%}$ & $\mathbf{1 8 \%}$ \\
\hline & 33 & $9 / 25$ & $5 / 23$ & $1 / 21$ & $0 / 3$ & $0 / 11$ & $15 / 83$ \\
\hline & & & & & & & \\
\hline $\mathbf{\Sigma}$ & Total & $\mathbf{2 4 \%}$ & $\mathbf{9 \%}$ & $\mathbf{1 1 \%}$ & $\mathbf{2 \%}$ & $\mathbf{3 \%}$ & \\
\hline & 366 & $57 / 241$ & $17 / 191$ & $22 / 196$ & $1 / 54$ & $4 / 117$ & \\
\hline
\end{tabular}

\section{Les caractéristiques du référentiel temporel négociatoire}

Les accords conclus ne signifient pas une communauté de vue à propos du temps de travail entre l'employeur et le salarié. Pourtant et c'est notre hypothèse, l'accord sur une forme temporelle suppose un accord sur une certaine représentation de la situation: le référentiel temporel qui accompagne la négociation constitue bien la condition d'un accord. Bien entendu ce référentiel élaboré dans un contexte précis, lié à la négociation,

\footnotetext{
${ }^{5}$ La figure peut se lire de la manière suivante: Le premier processus lié à la réduction de la durée du travail apparaît 61 fois dans notre échantillon. La CGT qui est présente dans 39 de ces accords, en refuse 6 . Son taux de refus est donc de $15 \%$.
} 
disparaît avec elle. Nous proposons d'explorer la dynamique de construction des accords pour caractériser ensuite ce référentiel négociatoire.

\subsection{La dynamique de construction des arrangements temporels}

Le sens des arrangements temporels ne peut être déduit mécaniquement des compromis négociés. D’abord parce que ces compromis désignent un résultat qui ne préjuge pas des manières de l'obtenir: différentes stratégies peuvent être utilisées par les négociateurs pour parvenir à un accord et ces stratégies peuvent varier d'un contexte à l'autre en fonction notamment de l'histoire sociale de l'entreprise. Ensuite, parce qu'un accord est un compromis provisoire qui ne signifie pas pour autant que la réalité codifiée dans l'accord est homogène d'une situation à l'autre, ni même que cette codification est partagée par tous.

Pour éviter de conclure un peu hâtivement à un changement des valeurs qui ordonnent les pratiques, il convient d'analyser les chemins par lesquels la négociation passe, pour comprendre tant la complexité et la fragilité des arrangements temporels, ainsi que la variabilité des stratégies qui ont été combinées pour les obtenir. C'est pourquoi nous avons procédé à une analyse monographique effectuée dans dix entreprises au travers de vingt entretiens (enregistrés et de 2 heures environ) avec les représentants de la direction et des organisations syndicales. Les entreprises ont été choisies, parce qu'elles avaient signé un accord sur le temps de travail en 1994 ou 1995 qui nous paraissait pertinent au regard des pivots structurants la négociation (la réduction de la durée du travail, modulation-annualisation, temps partiel et les horaires de travail). Sans qu'il soit possible d'exposer ici en détail les résultats, l'analyse de la dynamique de construction des arrangements temporels révèle la complexité de la négociation: tout d'abord la négociation est doublement finalisée (a), ensuite elle conduit à des solutions variables, c'est à dire à un cadre temporel éclaté et remettant en cause les repères temporels habituels (b), enfin (c) les chemins de la négociation reposent sur une pluralité de stratégies et sur la construction d'un compromis entre maintien de l'emploi et de la reprise d'initiative par l'employeur sur la question de l'emploi du temps du salarié.

\subsection{A La négociation sur le temps de travail est orientée vers une double finalité}

La négociation est orientée vers l'organisation de la production, plus que vers l'amélioration des conditions de vie au travail; ils visent à définir des règles temporelles permettant une souplesse d'organisation de la 
production en fonction des fluctuations de l'environnement: disponibilité exigée par le client, variations du marché ou du plan de charges, fluctuations saisonnières.

La négociation n'est pas seulement orientée vers une finalité externe, mais aussi vers une finalité interne, par exemple: un accord sur le temps partiel est construit dans une situation pour «créer un mouvement de signataires » en faisant de ce thème un objet mobilisateur favorisant l'émergence d'un nouvel acteur syndical pour s'affranchir du rôle hégémonique du syndicat dominant; dans une autre situation, il est construit pour diminuer le temps de travail des cadres estimé excessif par les intéressés en faisant de ce thème une occasion d'engager un débat sur la mesure du temps de travail des cadres. L'enjeu de la négociation dépasse le thème débattu: la négociation chemine dans un contexte marqué par les événements (grèves, conflits) et par un état des rapports de forces entre syndicats et direction, mais aussi entre syndicats eux-mêmes.

\subsection{B Solutions variables, cadre temporel éclaté et remise en cause des repères temporels habituels}

Les solutions envisagées pour atteindre l'objectif de flexibilité varient d'une situation à l'autre: du repos compensateur de remplacement pour limiter les heures supplémentaires, du temps partiel annualisé, de l'éclatement des deux jours de repos hebdomadaire consécutifs, du temps partiel et des horaires variables, la semaine de quatre jours comprenant le travail du samedi, une réduction du temps de travail pour faire face à la baisse du plan de charges, un recours au travail de nuit des femmes pour augmenter la disponibilité de la main-d'oeuvre. Cette variété de mesures conduit à un cadre temporel éclaté dont les composantes ne forment pas une vision cohérente, et ce d'autant moins que ce « temps en mosaïque » s'accompagne d'une remise en cause des repères temporels habituels. On passe (a) d'un repérage temporel hebdomadaire à un repérage annuel, (b) d'un repérage du repos de fin de semaine basé sur deux jours consécutifs à un éclatement et à une délocalisation de cette période de repos, (c) d'une limitation du travail de nuit à son extension aux femmes qui en étaient exclues, (d) d'un repérage en terme d'emploi à temps plein à une vision de l'emploi à temps réduit. La remise en cause des marqueurs temporels habituels est aussi une remise en cause des «normes temporelles du travail », voire des normes de travail.

\subsection{Diversité des stratégies, maintien de l'emploi et renforcement du contrôle}

Diverses stratégies peuvent déclencher ou accompagner la négociation sur le temps de travail. Dans un cas, le repos compensateur n'est obtenu qu'après l'intervention de l'inspection du travail, tandis que dans l'autre il 
est obtenu avec l'agrément des intéressés pour maintenir l'emploi. Dans deux autres situations, la « menace » de licenciements exprimée par l'employeur a infléchi les positions syndicales à l'égard du travail du samedi. Dans d'autres situations, l'un des syndicats organise une consultation du personnel qui révèle l'opposition des destinataires potentiels à toute mesure ayant une emprise sur sa vie hors de l'entreprise et qui fragilise la position des syndicats qui avaient l'intention de signer l'accord.

De plus les chemins de la négociation sur le temps de travail passent par le maintien (et non l'augmentation) de l'emploi qui est la monnaie de l'échange contre l'acceptation du renforcement du contrôle de l'employeur sur l'emploi du temps du salarié. En échange du maintien de l'emploi, l'employeur donne les moyens d'une part de reprendre l'initiative dans la fixation des périodes de travail et de disponibilité des salariés: le travail du samedi et l'annualisation du temps partiel illustrent cet aspect; d'autre part l'employeur se donne les moyens de contrôler plus directement la mobilisation des salariés par un usage différencié de la maind'oeuvre en fonction des besoins de l'entreprise.

\subsection{Le référentiel temporel qui accompagne la négociation}

Que nous apprend la négociation du temps de travail?

D’abord les négociations indiquent la capacité des acteurs de débattre sur une question essentielle qui les divise, avec pour les uns la nécessité de retrouver une «concordance des temps » et pour les autres la nécessité d'augmenter la flexibilité de la production. Entrer en négociation, c'est accepter de construire une espace de discussion pertinent pour débattre, dans un contexte précis, de la question de l'ordre temporel.

Ensuite ces négociations indiquent la capacité des acteurs à construire un cadre temporel de travail légitime: certes il s'agit de solutions communes qui n'ont pas la même force coercitive et rien ne préjuge des modalités de mise en oeuvre. Ce cadre temporel est le résultat de compromis, de concessions réciproques et de contreparties qui n'ont de sens et de portée que dans un contexte localisé: le cadre temporel a une cohérence locale. Enfin ces négociations indiquent que les parties en présence s'accordent sur un ordre temporel qu'ils s'engagent à respecter: le cadre temporel a une certaine robustesse, puisqu'il a été conçu pour

être mise en oeuvre. Bien entendu ce contrat est fragile: d'abord parce que tous les syndicats ne sont pas signataires; ensuite parce que l'usage de ce cadre nécessite son interprétation et son adaptation aux situations de ses destinataires.

En conséquence nous pensons que la négociation du temps de travail aboutissant à des accords produit un équilibre provisoire basé sur un ordre temporel en transition. Pour autant, il nous reste à explorer la solidité 
de cet accord: la négociation aboutit-elle à réduire l'asymétrie des représentations du temps de travail que véhiculent les différents acteurs?

La négociation conduit-elle à des accords reposant sur une représentation unique du temps de travail? Certainement pas. L'analyse des pratiques de construction du cadre temporel montre que ce cadre est conçu davantage pour favoriser la flexibilité de la production que pour améliorer les conditions de vie des salariés, même si la flexibilité est échangée contre le maintien de l'emploi. Il révèle la nature des compromis temporels élaborés. Cette analyse pourrait nous amener à conclure que les organisations syndicales sont prisonnières de cette logique tournée vers la flexibilité. L'étude monographique permet de douter de cette affirmation. Rien n'indique que les conduites syndicales obéissent à une conception du temps de la production abstrait et mécanique qui mène à une vision du temps vide de contenu. Les chemins qu'emprunte la négociation sur le temps de travail rappellent la dualité des rationalités en présence et des tensions entre les acteurs: un arrangement temporel ne s'impose pas mécaniquement, mais il est construit par confrontation, par négociation et conflit. C'est dire qu'on ne peut pas interpréter les compromis temporels comme des solutions temporelles exclusivement tournées vers la flexibilité de la production: les arrangements temporels dans leurs finalités, dans leur contenu ou dans leur mode de construction cristallisent cette dualité de logiques et cette tension entre les acteurs.

Comment interpréter la nature, la portée et la solidité des accords? Sur quelle vision du temps de travail reposent-ils? Notre hypothèse interprétative est que le sens d'un accord dépend du référentiel temporel construit par la négociation: il s'agit d'une composante fonctionnelle ou opératoire qui correspond à l'ensemble des représentations et des connaissances engagées et mobilisées dans l'action négociatoire sur le temps de travail. Il s'agit donc d'un référentiel de situation dans la mesure où c'est le contexte variable de l'action qui décide de son contenu.

Notre hypothèse qu'il faut considérer comme une piste de recherche, est qu'un accord sur le temps de travail repose sur un référentiel de situation opératoire commun dans lequel la flexibilité est échangée contre le maintien de l'emploi; en revanche un accord ne suppose pas que les parties en présence aient une vision commune du temps de travail, ou qu'ils partagent le même référentiel temporel de base: tout au contraire, il peut s'accommoder d'une différence dans la manière de concevoir le temps de travail, comme nous le montrerons à propos du référentiel de base.

L'analyse de la dynamique de construction du cadre temporel, nous a permis d'expliciter quelques caractéristiques du référentiel temporel commun de situation mobilisé dans et pour la négociation: ce 
référentiel ne retient que ce qui est pertinent pour cette action, n'engage qu'une partie des savoirs et des représentations. Il est situé dans un contexte local dont il prend les caractéristiques essentielles.

\section{Le référentiel temporel de base}

En revanche le référentiel détenu par les parties en présence que nous appelons le « référentiel de base »est bien plus vaste et correspond à tout ce qui a été accumulé au cours de l'expérience et à toutes les représentations du temps. Nous pensons d'illustrer cette hypothèse en prenant appuie sur des entretiens menées auprès des responsables des organisations syndicales d'une région du Sud de la France. De ces entretiens, nous avons tenté d'extraire les caractéristiques du référentiel temporel de base (a): la pression temporelle, (b) la stabilité des repères temporels et (c) la maitrise du cadre temporel constituent les principales caractéristiques de ce référentiel.

\subsection{Avoir du temps pour soi et du travail pour tous}

La première caractéristique du référentiel temporel de base est la revendication de disposer de «plus de temps pour soi ». Bien entendu le thème n'est pas nouveau: Rezsohazy (1986) montre dans son enquête menée auprès de 1636 personnes et répété en 1975 et 1980 que ce qui gène le plus les gens dans ce qu'ils voudraient «faire ou être », c'est le manque de temps, qui apparaît comme facteur le plus perturbant. De même dans une enquête menée en 1982 (cité par Sue, 1994, p.12) « le temps pour vivre »apparaît comme le premier centre de frustration des français. Ce manque de temps se retrouve dans l'enquête menée en 1992 au Canada auprès de 9815 personnes (cité par Pronovost, 1996, p.54): la réponse la plus retenue porte sur le sentiment de « ne pas avoir tout accompli, ce qu'on voulait faire dans la journée ». C'est dans ce climat de pression temporelle, que les organisations syndicales expriment leur point de vue qui peut se résumer par le propos de l'un d'entre eux: « plus de temps pour soi, du travail pour les autres ».

Cette expression se traduit, pour eux, par la nécessité d'une réduction de la durée du travail hebdomadaire, voir par la semaine des 4 jours pour avoir du temps pour soi.

«Il faut vraiment que la réduction soit forte, 35 heures, c'est peut être pas suffisant, ça doit être une étape pour aller plus loin. L'objectif visé aujourd'hui c'est bien 32 heures, la semaine de 4 jours, c'est ça () réduire le temps de travail c'est gagner du temps de repos; c'est du temps pour vivre, pour les loisirs pour être en famille ». (CGT) 
"Réduire l'importance ou l'amplitude de travail par un certain nombre de congés supplémentaires et parallèlement à cela, réduire aussi le temps de travail d'une façon hebdomadaire ou journalière et c'est ainsi donc que dans le processus, dans les revendications que nous avons mis en place, depuis longtemps, nous avons affiché notre nécessité de revenir à 35 h par semaine. » (FO)

«Donc nous on défend la semaine de 4 jours c'est peut être 4 fois 10 heures, je ne sais pas c'est suivant l'entreprise mais en disant; on est là présent 4 jours, mais au moins le cinquième jour, on est vraiment de repos, on est chez nous. Pour quelqu'un qui est cadre, il est clair qu'une demi-heure par jour, on la fera quand même. Parce qu'on est embauché pour faire un travail, donc puisqu'on est présent on le fera. (...) Je dirai travailler 4 jours sur 7 ça laisse 3 jours de loisir.» (CGC).

«Pour que la réduction du temps de travail soit efficace économiquement, il faut qu'il y ait 3 règles de mise en oeuvre: (1) massive, l'objectif c'est d'atteindre les 32 heures par une loi cadre. (2) multiple et (3) diversifiée pour tenir compte des différentes spécialités des entreprises. »(CFDT).

« Ramener la semaine à 37 h. pourquoi pas, d'une manière très impérative, mais modulée » (CFTC).

En revanche la question du partage du travail associée à une diminution de salaire divise les syndicats: trois entre eux pensent qu'elle est inéluctable. Pour les uns la perte salariale est acceptée: «Je dis qui dit partage dit aussi diminution du salaire, il faut en arriver jusque là » (CFTC).

"Après la difficulté, puis, ce sur quoi on tourne tout autour, sur la réduction du temps de travail, c'est le financement de la réduction du temps de travail et c'est là-dessus que d'ailleurs, on a des différends avec les autres organisations syndicales. Puisque les autres disent 32 heures sans réduction sans perte de salaire. Nous, on est un peu plus nuancé. » (CFDT).

«Je pense que la réduction du salaire elle joue, contre l'emploi, parce que moins de salaire, c'est moins de consommation moins de consommation c'est moins de débouchés pour l'entreprise forcement à un moment donne on est déjà dans cette difficulté où ils ont tellement réduit les salaires de partout »(CGT). 
"Quand on ne peut plus taxer les gens, c'est qu'ils sont à un tel niveau qu'ils ne peuvent. Voilà pourquoi, aujourd'hui, on ne peut pas établir une théorie de cette nature sans que pour autant, on regarde comment on l'applique. » (FO).

\subsection{Stabilité des repères temporels et retour sur la famille}

La deuxième caractéristique du référentiel temporel concerne la revendication d'une stabilité des repères temporels. Les régularités temporelles qui scandent la vie d'un individu (école, travail, retraite) sont remises en cause, notamment par des périodes de chômage auxquelles sont soumis bon nombre de personnes, mais aussi par les situations de précarité quelles rencontrent au cours de la vie professionnelle. A cela s'ajoute la dissociation croissante entre d'un côté le temps des machines et de l'autre le temps des personnes insérées dans des activités multiples.

La remise en cause des régularités est renforcée par la multiplicité des configurations temporelles que l'individu rencontre dans la vie de travail et par les dysharmonies temporelles que les configurations génèrent dans la vie quotidienne: les repères temporels habituels sont en quelque sorte brouillés. Par repères temporels on entend les marqueurs qui délimitent et rythment la vie professionnelle et qui déterminent le passage des activités professionnelles aux activités non-professionnelles. Sue (1996) parle de «dilution des repères temporels »: Aujourd'hui, dit-il, ces repères sont brouillés: annualisation, temps partiel, travail de nuit et de fin de semaine.

C'est dans ce climat de brouillage des repères temporels que s'expriment les positions des acteurs syndicaux à l'égard des mesures temporelles permettant l'annualisation du temps de travail, le travail à temps partiel, le travail de nuit des femmes. Une première illustration de cette revendication de stabilité des repères temporels nous est donnée par les réactions à l'égard de l'annualisation du temps de travail qui est considéré en référence à la famille et la division du travail qui s'y opère selon les sexes.

«Alors l'annualisation, bien entendu, c'est un problème tout à fait différent et pour lequel, on ne peut pas être d'accord, parce que l'annualisation, il y a une question aussi de qualité de vie, l'annualisation veut dire simplement la destruction de la cellule familiale. Ca veut dire aujourd'hui qu'un chef d'entreprise va vous dire dans le cadre de l'annualisation: Vous allez pouvoir travailler en fonction du travail que j'ai. Et ça peut déjà aller par rapport au congé, parce qu'il n'est pas impossible qu'il y ait une commande pendant la période de congé et, comme il a de mai à octobre pour vous les donner (les congés), rien ne lui interdit de me donner en mai ou en octobre. Ca serait quand même pas intéressant que le père prenne 
le vacances en octobre, que les enfants les prennent en juillet et août et que la mère le prenne au mois de mai, c'est déjà la première des choses », (FO).

"C'est la vie familiale qui est mise en cause, la mère de famille qui a des enfants, elle ne pourra pas bloquer son temps de travail sur ce niveau de l'année et puis qu'est ce qu'elle va faire les autres mois, elle aura un temps de travail beaucoup plus long. Comment elle pourra faire pour garder ses enfants? Il y a un équilibre à respecter, je pense, il y a un cadre, mais ce cadre a des limites. Ces limites à mon avis qu'il ne faut pas dépasser» (CFTC).

"Alors ça devient facile quand, c'est le vendredi soir, vous avez fini la semaine, vous êtes prêt à partir, le client vient de téléphoner et lundi matin, il lui faut 53000 trucs devant la porte, parce qu'il a ses trucs arrêtés et qu'il faut qu'il travaille et on nous dit: Oui, il va falloir travailler samedi, il va falloir travailler dimanche et si vous ne le faites pas, il ne va pas être livré, s'il n'est pas livré, il va nous enlever la commande et s'il nous enlève la commande, on n'aura pas de travail pendant 6 mois (...)

C'est du cinéma ça, moi je dis qu'au chantage, il ne faut jamais céder, si vous mettez le doigt une fois, c'est fini pour la vie, vous allez travailler tous les samedi, tous les dimanche et tout le temps, il y aura toujours ce truc, un moment donne, vous pouvez avoir cette idée, vous dire, effectivement c'est vrai, puis après vous allez vous rendre compte que c'est quelque chose d'exceptionnel et avec le temps qui passe, ça devient le normal, et on s'en sort plus »(CGT).

Un autre thème, celui du travail de nuit et du week-end permet d'illustrer cette quête de stabilité des repères temporels.

«Au niveau du travail de nuit, du travail le week-end, là nous avons une approche qui peut être n'est pas la même pour tout le monde. Nous sommes viscéralement opposés à ces formules là, mais nous sommes très attentifs à la mise en place de ces réformes, parce que compte tenu du fait que nous représentons, nous défendons des valeurs familiales, une approche familiale de la société, ce type de travail... le week-end ou les nuits ne favorise pas la vie de famille et nous ne sommes pas opposes comme ça, il y a des endroits où on ne peut pas faire autrement, mais notre approche n'est quand même pas de généraliser ce type de travail. 
Nous sommes plutôt pour une modulation du temps de travail le reste de la semaine, mais pas le week-end et le samedi. (...). Nous avons toujours été opposés et notre syndicat répond systématiquement non, aux ouvertures des grandes surfaces les week-ends... Nous n'aimons pas ces approches là...Il y a une approche travail société qu'il faut quand même garantir, une symbiose entre les deux qui fait que si on privilégie trop le travail, c'est la cellule familiale qui se détruit, c'est aussi beaucoup de problèmes qui sont générateurs aussi de chomage... Si vous voulez, nous avons plus une approche humaniste des choses, qu'économique tout en sachant que dans certains endroits, peut être c'est indispensable.(...). Le travail n'est pas un but en soi, il permet de vivre, de vivre correctement, mais si on fait du travail, si on s'amuse à travailler le samedi et le dimanche, si on donne des dérogations à ce genre de choses, c'est une conception de vie qui disparaît, c'est la cellule familiale qui à terme sera détruite, et je pense, à mon avis, nous c'est pas la meilleure approche. Je pense qu'il y a l'autre formule, de l'aménagement du temps de travail, sans en arriver là (CFTC).

\subsection{Maîtriser l'usage du cadre temporel de travail et préserver son emploi}

La troisième caractéristique du référentiel de base concerne la maitrise du cadre temporel des activités professionnelles. A l'insuffisance de temps pour soi et à la volonté de disposer de repères temporels stables et cohérentes avec d'autres temps sociaux, les responsables syndicaux ajoutent la nécessité de maitriser l'usage du cadre temporel du travail. La question ne porte pas sur le temps en soi que l'on pourrait traduire en termes de conflit entre l'aliénation par le travail et la jouissance par le temps libre, pour reprendre l'expression de Sue (1994), mais sur le caractère imposé des nouvelles règles temporelles. Non seulement ces nouvelles règles détruisent les repères temporels habituels, mais elles renforcent le contrôle de l'emploi du temps des salariés, de disponibilité et de leur mobilisation. Au contrat de subordination du salarié à son employeur qui est inscrit dans le contrat de travail, s'ajoute un contrat de soumission à la gestion de l'emploi du temps du salarié par l'employeur, notamment pour maintenir son emploi ou par crainte de le perdre.

A propos de l'annualisation, le responsable CFDT observe que «les gens disent que, si ça continue comme ça, tout va partir à l'étranger et on ne va pas avoir notre boulot ». De même le responsable CGC déclare: «Je pense que c'est assez intéressant et ça évite des licenciements ou des contrats à durée déterminée ». A propos du temps partiel, les responsables syndicaux sont clairs. «Le travail à temps partiel, je pense qu'il faut que ça soit inscrit dans les accords d'entreprise mais que ce soit temps partiel voulu et non imposé c'est 
le $1^{\circ}$ point, $2^{\circ}$ point que ce ne soit pas un frein à la carrière, on doit avoir un suivi de la carrière de ces gens là. Si on l'a négocié, l'entreprise était d'accord pour le mettre en place donc il faut le suivre. Donc on est favorable au temps partiel. »(CGC).

Enfin pour le travail de nuit, il est accepté sous contrainte: "S'il n'y a pas de contrainte réelle et forte, je dis de façon naturelle, les gens, n'ont pas envie de travailler la nuit. (...). Vous savez, tout se traduit en terme économique. Ce qui est bien la démonstration que si les gens gagnaient suffisamment pour s'éviter la prime de nuit et le week-end ils ne feraient pas. L'attrait qui a été fait. Ce n'est pas, ce n'est jamais une volonté réelle, c'est un attrait économique. (...). Mais, si pour le même salaire il fallait choisir entre le jour et la nuit, faites-moi confiance qu'ils ne le feraient pas. » (FO)

«Sur le travail posté c'est pareil, j'en connais qui sont organisés, avec leur époux, c'est pas une vie familiale, un qui travaille la nuit, l'autre qui travaille le jour, on se laisse des petits mots sur la table, mais ça règle un tas de problèmes, tels la garde des enfants, économie des sous de crèche, parce que ça répond à des difficultés, 33\% de primes et puis le pouvoir d'achat. (...). Il y a celui qui cherche du travail, il arrive chez l'employeur, il a un contrat devant lui, le travail est posté. Si le type est au chômage, il ne va pas commencer à débattre avec l'employeur sur le travail posté. Dans sa tête ça fait six mois qu'il galère, il signera, c'est normal. Après il y a celui qui a des difficultés. 33\% de salaire en plus ça l'arrange, parce que ça va lui permettre de partir en vacances, un peu plus pour les gosses. (...). Je ne porte pas de jugement làdessus. Je ne suis pas dans cette situation. Je suis dans la situation de l'activité revendicative, sur des objectifs précis et je reste là dessus. » (CGT)

\section{Discussion et conclusion}

Nos recherches ont tenté premièrement de montrer que le temps de travail constitue une réalité socialement construite: il constitue bien le cadre temporel préexistant aux activités professionnelles et visant à les ordonner. Pour autant, ce cadre temporel de travail ne peut être considéré comme une réalité objective, détachée des individus et s’imposant « mécaniquement » voire « naturellement» aux personnes. Ce cadre est le résultat d'une activité de négociation débouchant sur la production d'un ordre temporel des activités professionnelles. Cet ordre temporel est variable, notamment du fait qu'il est en permanence renégocié: il est établi par convention en fonction de choix qui dépendent des conceptions socioculturelles des négociateurs et 
des rapports de forces que chacun des protagonistes tente de tirer à son avantage pour faire valoir ses conceptions du temps et contrôler le modèle rationalisateur du temps de travail.

Deuxièmement nous avons montré que l'ordre temporel des activités professionnelles en tant que construction négociée n'obéit pas à une seule rationalité qui est celle de la production, mais que l'ordre temporel porte aussi la marque de la rationalité de la vie quotidienne. L'arrangement temporel incorpore l'ambivalence de la notion de temps de travail qui désigne deux réalités bien différentes: d'un côté la réalité du temps déterminant et mesurant de manière abstraite les activités professionnelles en vue d'une performance productive; de l'autre la réalité du temps qualitatif, renvoyant aux activités concrètes, appréhendé de manière personnelle, visant à ordonner de manière singulière par les sujets agissants et visant à ordonner leurs activités en vue d'obtenir une certaine cohérence et concordance dans leur temporalités. La négociation ne réussit en aucune manière à transformer le temps qualitatif et subjectif en un temps quantitatif et objectif: elle rend possible la construction du temps de travail comme temps hétéronome, c'est à dire comme cadre temporel dans lequel les activités professionnelles sont inscrites. Que ce cadre ait pu être négocié ne doit pas laisser croire qu'il est contrôlé par la personne qui travaille; dès lors que «le temps prévaut sur la tâche » selon l'expression de Naville (1963), l'homme qui perd la maîtrise du temps et le contrôle de son processus d'action, est affaibli. Cette réalité largement analysée par Friedmann (1950) pour qui la perte du contrôle de la personne qui travaille sur son propre temps et sur son travail, conduit à une « déshumanisation ».

Troisièmement nous avons tenté de montrer que les arrangements temporels ne reposent pas sur une conception commune du temps de travail. La négociation sur le temps de travail mobilise des savoirs et des représentations constitutifs de ce que nous avons appelé les référentiels temporels. Notons que les référentiels temporels de situation mobilisés par les organisations syndicales dans la négociation se distinguent les uns des autres. La comparaison intersyndicale détaillé de ces référentiels mettrait en relief qu'une position commune de l'acteur syndical face au problème posé reste hypothétique, même si comme nous l'avons vu des alliances de situation sur des thèmes précis restent possibles.

Nous avons montré que les pratiques temporelles analysées au travers la construction négociée du cadre temporel sont orienté par un ou des référentiels temporels: il s'agit de représentations, de croyances, de valeurs, des savoirs et des connaissances acquises. Les pratiques de négociation ne témoignent pourtant pas d'une traduction mécanique du référentiel: autrement dit le référentiel temporel ne détermine pas totalement les pratiques, mais les oriente. En effet les pratiques de négociation conduisant à des accords sur le temps de 
travail pourraient laisser croire à une sorte de convergence entre les employeurs et les salariés à propos du temps de travail. Pourtant l'analyse de ce que nous avons appelé le référentiel de base révèle que les valeurs énoncées par les organisations syndicales ne visent pas la flexibilité de la production, mais sont orientées vers la vie du salarié: du temps pour soi, des repères temporels habituels pour favoriser la synchronisation des temporalités, de la maîtrise du cadre temporel pour maintenir son emploi. C'est pourquoi il nous semble que ce qui explique la rationalité des pratiques de négociation, c'est la production par les acteurs concernés d'un référentiel qui prend en compte les données du contexte (rapports de forces, situation de travail, de l'emploi, menaces, demandes des salariés) et qui mobilise les représentations de base. L'accord sur le temps de travail signifie qu'il y a un accord de forme entre employeur et salarié tant sur la solution temporelle choisie que sur le référentiel temporel construit pour et par la négociation. Cela signifie aussi que l'accord renferme un désaccord, car il n'y a pas d'accord de fond sur une vision commune du temps de travail, bref un accord de forme cohabite avec un désaccord de fond.

Il convient cependant de s'interroger sur la nature du référentiel temporel de base qui met en avant la nécessité du temps pour soi, qui marque un retour à la famille qui valorise la synchronisation des temporalités. D'une part il conviendrait d'explorer la diversité de ces conceptions du temps d'un groupe à l'autre, d'une société à l'autre. D'autre part il faudrait interroger les conceptions du temps du point des visions sexuées qui traversent la société.

\section{Bibliographie}

Friedmann G. (1950).- Ou va le travail humain? Gallimard, Paris.

Grossin W. (1984).- «Les temps de travail », in: De Coster M. et Pichault F. (Eds), Traité de Sociologie du Travail, de Boeck, Bruxelles, pp.127-144.

Mercure D. (1995).- Les temporalités sociales, L'Harmattan, Paris. de décembre 1993. Rapport final, Toulouse, LIRHE-CERTOP, Juin 1996, 180 p.

Morin M.-L., Terssac (de) J., Sublet S., Thoemmes J. (1996).- L'évaluation du volet temps de travail de la quinquennale de décembre 1993. Rapport final, Toulouse, LIRHE-CERTOP, juin 1996, 180 p. 
Naville P. (1963).- Vers l'automatisme social, Gallimard, Paris.

Naville P. (1969).- préface de Grossin W. (1969), Le travail et le temps, Anthropos, Paris.

Pronovost G. (1996).- Sociologie du temps, de Boeck, Bruxelles.

Soupiot A. (1995).- « Temps de travail: pour une concordance des temps », Droit Social, no 12.

Sue R. (1994).- Temps et ordre social, P.U.F, Paris.

Thoemmes J., Terssac (de) G. (1995).- La construction des arrangements temporels: Une étude de cas sur 11 ans, Sixième Séminaire International sur le Temps de Travail (SITT) à Blankenberge, Belgique. 